\title{
Unusual Penetrating Head Trauma Gallsed by Sickle in Farmers
}

\section{JCR}

\section{Nandesh Kumar', Rohił Kumar ${ }^{2}$}

Departments of 'Surgery and ${ }^{2}$ Neurosurgery; Patna Medical College, Patna, Bihar, India.

\section{Abstract:}

Penetrating head injuries are rare in farmers but can be a significant cause of morbidity. We report a case of a fourteen-year-old farmer boy who sustained a fatal trans-orbital penetrating injury by sickle. The sickle was lodged intracranially with its tip close to the cavernous sinus, damaging the optic nerve. This case highlights the vulnerability of farmers to penetrating head injury by farming equipments and as well as the necessity for multidisciplinary approach to prevent mortality and morbidity in such cases.

Key words: Agriculture, Craniocerebral Trauma, Farmers, Head Injuries, Morbidity.

\section{Introduction}

Although head injuries are a common result of accidental trauma in all age groups, penetrating head trauma is less common. We report a case of a 14-yr-old farmer boy who sustained penetrating injury by a sickle (a manually harvesting device) highlighting the mode, circumstances, site of injury, resultant damage to vital structures and treatment outcome.

\section{Case Report}

A 14 year old boy was brought to the surgical emergency room after sustaining a penetrating injury in the vicinity of left eye due to fall over a sickle held in his hand. He reached emergency room four hours after injury while the sickle was still inside the wound. At the time of admission, patient was conscious, oriented to time, place and person but bit drowsy. General examination revealed the inner margin of left orbit was penetrated by the sickle. The point of entry was just superomedial to the eyeball [Fig.1]. The patient was holding the hanging portion of sickle in his hand to avoid pain. Ocular examination revealed diminished visual acuity with slight restriction of medial gaze of left eye. The left pupil was dilated and fixed with slight non pulsating proptosis. Detailed neurological examination revealed a normal mental status. The patient had no sensory or motor deficit and plantar responses were flexor bilaterally. A computed tomography (CT) scan demonstrated a metallic foreign body passing between the medial side of the left eye ball and lateral wall of nose and ethmoid air cell, passing posteriorly through the left optic canal to the middle cranial fossa. The tip of the foreign body was seen to pierce through the left greater wing of the sphenoid and reaching into the middle cranial fossa. The tip was located about

Corresponding Author: Dr. Nandesh Kumar

Email: nandyuc@gmail.com

Received: March 25, 2016 | Accepted: June 29, 2016 | Published Online: September 10, 2016

This is an Open Access article distributed under the terms of the Creative Commons Attribution License (creativecommons.org/licenses/by/3.0)

Conflict of interest: None declared | Source of funding: Nil | DOl: http://dx.doi.org/10.17659/01.2016.0104 
$1 \mathrm{~cm}$ lateral to the left cavernous sinus. The sickle appeared to be just around the left optic foramina possibly damaging optic nerve. The lesion appeared to involve laceration of the temporal lobe of left cerebral hemisphere. The left lateral rectus muscle was also likely involved by the lesion. The length of the intracranial part of the sickle was about 8 $\mathrm{cm}$ [Fig.2]. Bilateral maxillary sinus sphenoid sinus, frontal sinus and bilateral ethmoid sinus appeared normal. Alveolar process of maxilla, petrous and squamous part of temporal bone were normal. A three centimetre curved incision centred over entry point of sickle was given for right small superomedial orbitotomy. Intracranial part of sickle was removed under direct visualization from the entry point with marked initial resistance [Fig.3]. No significant haemorrhage was found intra-operatively.

The wound was adequately debrided. The ethmoid bone and shredded galea were repaired. Ophthalmological and ENT opinion was sought from respective specialists. Postoperative brain CT revealed no residual intracranial hematoma along the penetrating path. Postoperative course was uneventful. Patient was mentally alert with no CSF leak. However ptosis of left eye and restricted movement of eye ball was noted. There was no improvement in visual acuity of left eye. Pupil was still dilated and fixed. Repeat brain CT done on fifth day did not reveal any additional finding. Mild wound infection was treated with appropriate antibiotics. Patient was then referred to ophthalmologist for further management of resultant visual defect.

\section{Discussion}

Penetrating head injuries have higher mortality and morbidity than blunt trauma even in civilian set up. Statistical data suggest that case fatality rates are higher for penetrating than closed injuries for all GCS, gender, age, and cause of injury categories. Transorbital penetrating injury are as such uncommon form of injury. These are potentially

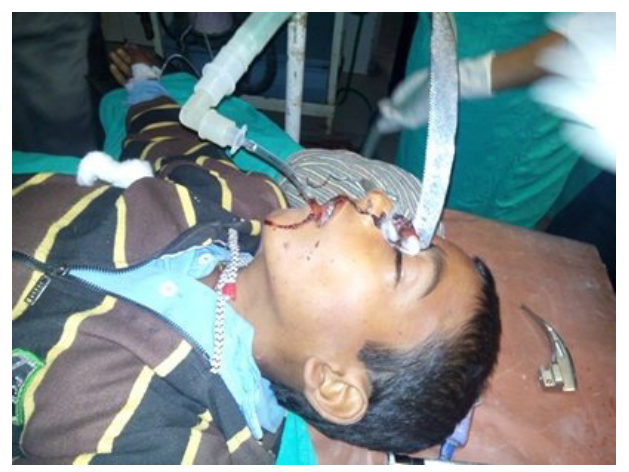

Fig.1: Pre-operative image showing sickle entry.

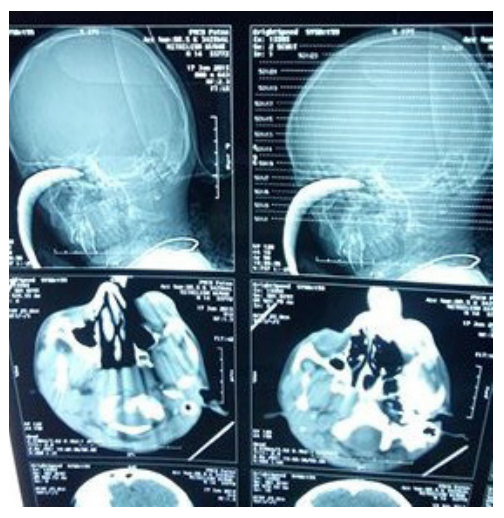

Fig.2: Pre-operative CT scan.

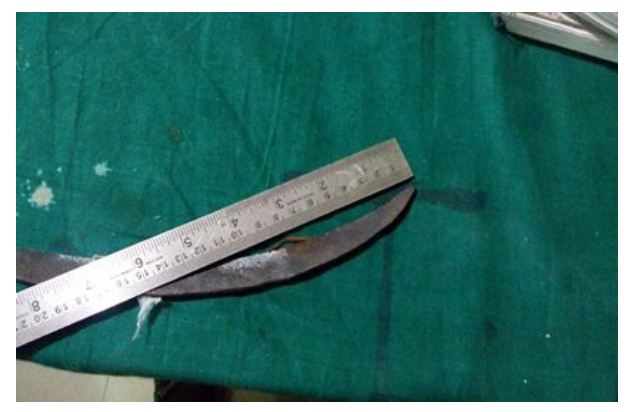

Fig.3: Removed sickle from cranium.

fatal and require a multidisciplinary approach. No attempt should be made to remove the foreign body without backup of an operating room because of the possibility of the object might be tamponading an injured vessel. A careful planning and a strict adherence to basic perioperative principles can 
lead to a satisfactory outcome [7]. Most common sites for entry wound are the temporal area and orbit where the bone is relatively thin. Foreign bodies typically penetrate the orbit from the medial canthus and usually pass through the optic canal and superior orbital fissure to lodge in the ipsilateral or contralateral side of the cranium. The most frequent path of penetration is via the roof of the orbit due to the fragile structure of the superior orbital plate of the frontal bone, often resulting in frontal lobe contusion. The second most frequent path of penetration is the superior orbital fissure, by which foreign bodies occasionally reach the brain stem through the cavernous sinus and cause serious damage [6]. The appropriate management in the peripheral setup is to leave the transorbital object in situ and transport the patient to the trauma centre carefully.

Management of patients with transorbital brain injuries and foreign bodies in situ should follow basic surgical principles, including removal of the object under direct vision in order to reduce further brain tissue damage by the foreign bodies catching on bone fragments. Following removal of the foreign body, thorough debridement with removal of all involved skull bone and foreign materials, hematoma evacuation followed by careful haemostasis along the trajectory, and meticulous dural closure to reduce the possibility of cerebrospinal fluid fistula are mandatory. For patients with deep-seated bullet in the brain it is not necessary to remove the bullet because of the potential risk for further brain damage and because of the low incidence of CNS infection. However, metal bars and other missiles with extra cranial components must be removed, and prophylactic antibiotics should be considered. $\mathrm{CT}$ is typically the first-line radiologic examination in the emergency room for head-injured patients. However, scanning artefacts from metal objects can sometimes limit visualization of brain tissue and brain damage along the trajectory of the penetrating object. In patients with transorbital brain injuries, the path of penetration often passes near the cavernous sinus and basal cisterns. Thus, corresponding brain contusion, haemorrhages with great mass effect may be obscured in the initial CT scan. We therefore recommend an immediate follow-up CT scan postoperatively in order to look for missed contusion brain damage and possible haemorrhage. An alternative approach is endoscopic brain surgery via the transorbital route, which may offer direct visualization of unidentified brain damage along the penetrating path and provide direct haemostasis.

Kieck and Villiers reported 11 vascular lesions identified with cerebral angiography in 18 transorbital head-injured patients (61\%). However, due to prolonged radiologic intervention procedures and inadequate CT scan resolution, patients with extended transorbital brain injuries may deteriorate rapidly. Hung et al. proposed that prompt craniotomy for decompression and direct haemostasis for vascular injuries cerebral, angiography in patients with extended transorbital brain injuries as better option than cerebral angiography in patients with extended transorbital brain injuries.

\section{Conclusion}

Accidental penetrating head injuries can be an important cause of disability among the children of farmers especially in the rural areas working under situations with improper safety and with life-threatening instruments. Transorbital brain injury caused by a sickle is a rare but fatal event. Emergent surgical intervention and early followup CT is necessary if there is to be any chance of saving the life of the patient.

\section{References}

1. Harsh GR. Penetrating wounds of the head. In: Wilkins RH, Rengachary SS, eds. Neurosurgery. New York: McGraw-Hill, 1985:1670-1678. 
2. Matsumoto S, Hasuo K, Mizushima A, Mihara F, Shirouzu T, Masuda K. Intracranial penetrating injuries via the optic canal. Am J Neuroradiol. 1998; 19:1 163-1165.

3. Davis GA, Holmes AD, Kulg GL. Delayed presentation of transorbital intracranial pen. J Clin Neuroscience. 2000;7:542-560.

4. Peek-Asa C, McArthur D, Hovda D, Kraus J. Early predictors of mortality in penetrating compared with closed brain injury. Brain Inj. 2001; 15:801 810.

5. Faraji M, Ashrafzadeh, Farah. Penetrating head injuries in children. Neurosurgery Quarterly.
2005; 15:160-163.

6. Schreckinger $M$, Orringer D. Transorbital penetrating injury: case series, review of literature and proposed management algorithm. J Neurosurgery. 2011;114(1):13-16.

7. Arslan $M$, Eseoglu $M$, Güdü BO, Demir I. Transorbital orbitocranial penetrating injury caused by a metal bar. J Neurosci Rural Pract. 2012;3(2):178-181.

8. Borkar SA, Garg K, Garg M, Sharma BS. Transorbital penetrating cerebral injury caused by wooden stick. Childs Nerv Syst. $2014 ; 30(8): 1441-1444$. 